\title{
The persistent puzzle of the geographic patterning of cardiovascular disease
}

\author{
Ana V. Diez Roux \\ Center for Integrative Approaches to Health Disparities, University of Michigan, 109 Observatory Street, SPH Tower 3671, Ann Arbor MI 48109, USA
}

It has long been known that health is geographically patterned (Jones and Moon, 1987). In fact, "place" has been a key dimension of epidemiology since its origins. Descriptive epidemiology emphasizes differences in disease distribution across places because it may provide important etiologic clues. Cardiovascular disease mortality, and in particular coronary heart disease (CHD) and stroke, vary markedly across and within countries (Lloyd-Jones et al., 2009). In the United States, there are up to two-fold differences in stroke and CHD mortality across states (Lloyd-Jones et al., 2009). Yet our ignorance about the reasons for these differences is surprising, especially given our knowledge of major heart disease and stroke risk factors. Together with the causes of trends over time - topic of debate and research in itself - the causes of geographic variations remain one of the big unanswered questions in cardiovascular disease epidemiology.

In this issue of Preventive Medicine, Howard et al. (G.C.M. Howard, Prineas R., Howard VJ., Moy CS, Sullivan LM, D'Agistino RB, McLure LA, Pulley LV, Safford MM) use unique data collected as part of the Reasons for Geographic and Racial Differences in Stroke (REGARDS) study, a national study of geographic variations in stroke, to investigate the extent to which established heart disease and stroke risk factors explain geographic variations in stroke and heart disease mortality. They construct summary aggregate measures of the Framingham Coronary Heart Disease Risk Score and the Framingham Stroke Risk Score for US states and investigate correlations between scores and corresponding mortality rates, between stroke and heart disease scores, and between stroke and heart disease mortality rates. As expected, each of these scores is positively associated with the corresponding mortality rate at the state level, with stronger correlations observed for CHD than for stroke. They find that CHD and stroke mortality rates are not correlated, suggesting that the etiologic processes leading to each outcome are distinct and could involve different sets of factors. In contrast the Framingham heart and stroke risk scores are positively correlated with each other. From this pattern of results (weak associations between CHD and stroke mortality but strong associations between scores) the authors infer that the risk factor components of the FRS do not explain the geographic variation in store and CHD mortality rates.

The correlational results reported by Howard et al. are compatible with the possibility that geographic differences are not explained by risk factors, but they also have limitations. If risk factors are important contributors to mortality differences, the geographic patterning of risk factor scores should mirror the geographic patterning of mortality

E-mail address: adiezrou@umich.edu. rates, and hence the ecologic correlations between stroke and CHD scores should be similar to the ecologic correlations of stroke and CHD mortality rates. But since the scores are imperfect predictors of the rates and both scores have components in common, it is predictable that the scores may be correlated even if the mortality rates are not. A correlation in the scores in the absence of correlation in the rates does not necessarily imply that risk factors do not contribute to geographic differences.

As noted by Howard et al., correlational analyses of ecologic data also raise a number of other methodological problems that limit the kinds of inferences that can be drawn. These data do not allow the authors to directly investigate how risk factors contribute to geographic differences by estimating state or regional differences in CHD or stroke mortality before and after controlling for risks factor data in individual-level analyses. This will however, become possible in the on-going REGARDS study which may provide the appropriate data as follow-up of the cohort continues (Howard et al., 2005).

Even under ideal circumstances, with individual-level longitudinal data, the investigation of the contributions of risk factors to differences in disease incidence or mortality is challenging. Many risk factors are measured with substantial error, or may not be measured for the full time period relevant to disease development (which is often the whole lifecourse). The observed contribution of risk factors to disease may be attenuated by these measurement problems and by not fully accounting for synergisms between risk factors - if they exist. These issues often complicate the interpretation of residual differences after adjustment for risk factors. Despite these methodological challenges, investigating to what extent risk factors contribute to the observed patterning remains an important goal as it can help identify new causal factors, and point to interventions to reduce spatial inequities.

A limitation of the summary risk factor scores employed by Howard et al. is that they do not include other factors, such as diet and physical activity. These behavioral factors are notoriously difficult to measure but could be important predictors of outcomes independently of the Framingham Risk Score components, and may be spatially patterned for a variety of social and economic reasons (Hahn et al., 1998). Environmental factors, such as air pollution, could also be involved in the geographic patterning if they prove to be causally important to CHD and stroke mortality (Brook et al., 2004). Although less emphasized in research of the geographic patterning of cardiovascular disease, psychosocial factors may vary over space for social or cultural reasons and could also contribute to geographical patterns in CHD or stroke. The probable involvement of a multiplicity of interacting factors will require studies that include measures of a 
variety of relevant domains and that fully capture geographic variability. The dearth of studies that allow detailed testing of the multiple factors hypothesized to be involved is an important limitation in the field as noted by Howard et al. The REGARDS study will contribute to filling this research gap.

If we were able to identify all the key proximal risk factors for CHD and stroke (as well as any interactions that are relevant) we would be able to fully "explain" any geographic variation observed, i.e. the geographic variation could be shown to be purely a function of the distribution of these factors. But this begs the question of why these risk factors are themselves geographically patterned to begin with. For example, if geographic variations in stroke are in part or wholly a function of the geographic patterning of diet, physical activity, and smoking, why do these factors vary over space? Ultimately, understanding the fundamental causes of geographic differences will likely require studying distal geographically patterned antecedents operating over the lifecourse, which will most likely be social or environmental in nature. This will require understanding the relevant social and environmental constructs, measuring them and empirically testing their effects.

Too often, we as researchers focus on narrow and tractable research questions. There are good reasons to this, but it also means that we sometimes avoid the more complex big picture questions in population health, such as the as yet unexplained and often debated geographic patterning of cardiovascular disease. In the analyses reported in this issue, and more generally in the broader REGARDS study, Howard and collaborators tackle this important unanswered question. Discovering the causes of these large unexplained geographic variations, will provide key insights not only into better strategies to prevent CHD and stroke, but, more generally, into the multilevel causes of cardiovascular disease.

\section{References}

Brook, R.D., Franklin, B., Cascio, W., et al., 2004. Air pollution and cardiovascular disease: a statement for healthcare professionals from the Expert Panel on Population and Prevention Science of the American Heart Association. Circulation 109 (21), 2655-2671.

Hahn, R.A., Heath, G.W., Chang, M.H., 1998. Cardiovascular disease risk factors and preventive practices among adults-United States, 1994: a behavioral risk factor atlas. Behavioral Risk Factor Surveillance System State Coordinators. MMWR CDC Surveill Summ 47 (5), 35-69.

Howard, G.C.M., Prineas, R., Howard, V.J., et al., Advancing the hypothesis that geograohic variations in riks factors contribute relatively little to observed geographic variations on heart disease and stroke mortality. Prev. Med.

Howard, V.J., Cushman, M., Pulley, L., et al., 2005. The reasons for geographic and racial differences in stroke study: objectives and design. Neuroepidemiology 25 (3), 135-143.

Jones, K., Moon, G., 1987. Health, Disease, and Society: A Critical Medical Geography. Taylor and Francis, London.

Lloyd-Jones, D., Adams, R., Carnethon, M., et al., 2009. Heart disease and stroke statistics-2009 update: a report from the American Heart Association Statistics Committee and Stroke Statistics Subcommittee. Circulation 119 (3), 480-486. 